\title{
VI. The calculation of radiation emitted in gaseous explosions from the pressure-time curves
}

\section{Major W.T. David M.A. M.Sc.}

To cite this article: Major W.T. David M.A. M.Sc. (1920) VI. The calculation of radiation emitted in gaseous explosions from the pressure-time curves, Philosophical Magazine Series 6, 39:229, 66-83, DOI: $10.1080 / 14786440108636017$

To link to this article: http://dx.doi.org/10.1080/14786440108636017

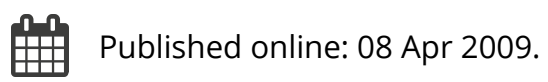

Submit your article to this journal ¿

Џ Article views: 6

Q View related articles ๘ 
symmetry) was uniquely privileged. But from the present, more general point of view there is certainly no reason for rejecting a meridian plane as the reference plane $(i=0)$, and this, i.e. any such reference plane, will precisely give, in addition to $(28 \cdot 1)$ the new coefficient $\left(28^{\circ} 1^{\prime}\right)$ and thus also new lines or components.

Still, the asymmetric nucleus will be richer in possible lines or components. Which of these are to be rejected, can be decided only on the ground of spectroscopic experience. One might put forward, for instance, as a restricting criterion that out of the three $\gamma$-values $(30 \cdot 2)$, that only is to be retained to which, cateris paribus, corresponds the greatest $W_{s}$, or perhaps the smallest $W_{s}$, or what not. Bat all such guesswork, unsupported by observation, would be pretty useless.

The chief purpose of the present paper has been to show the numerous and broad possibilities opened up by a nonspherical nucleus both with regard to the fine structure of lines or groups and to the very type of the spectrum series. All numerical applications of the general formulæ and their discussion in connexion with experimental spectroscopic knowledge are necessarily postponed to a later opportunity.

Research Dept., Adam Hilger.

London, October 18, 1919.

VI. The Calculation of Radiation emitted in Gaseous Explosions from the Pressure-Time Curves. By Major W. T. DAvid, M.A., M.Sc.*

\section{INTRODUCTION.}

1. T $\mathrm{N}$ this paper an attempt is made to build up formulæ by means of which the radiation emitted in explosions of inflammable mixtures of coal-gas and air may be calculated from the pressure-time curves. A large number of photographic films on which were traced curves of pressure and of radiation emitted $†$ have been examined, and it has been found possible to establish some simple equations which would seem to apply within fairly wide limits of mixture strength, density, and volume.

* Communicated by the Author.

+ Curves taken from a considerable number of these films have previously been published in the Phil. Trans. (A. vol. ccxi. pp. 375-410). Reference should be made to this paper for full details of the experimental methods employed. 
2. It will be convenient to describe here very briefly the experimental arrangements under which these films were taken. The gaseous mixtures were exploded in a cylindrical cast-iron ressel $30 \mathrm{~cm}$. in diameter and $30 \mathrm{~cm}$. in length (shown in fig. 1). The pressures of the gaseous mixtures

Fig. 1.

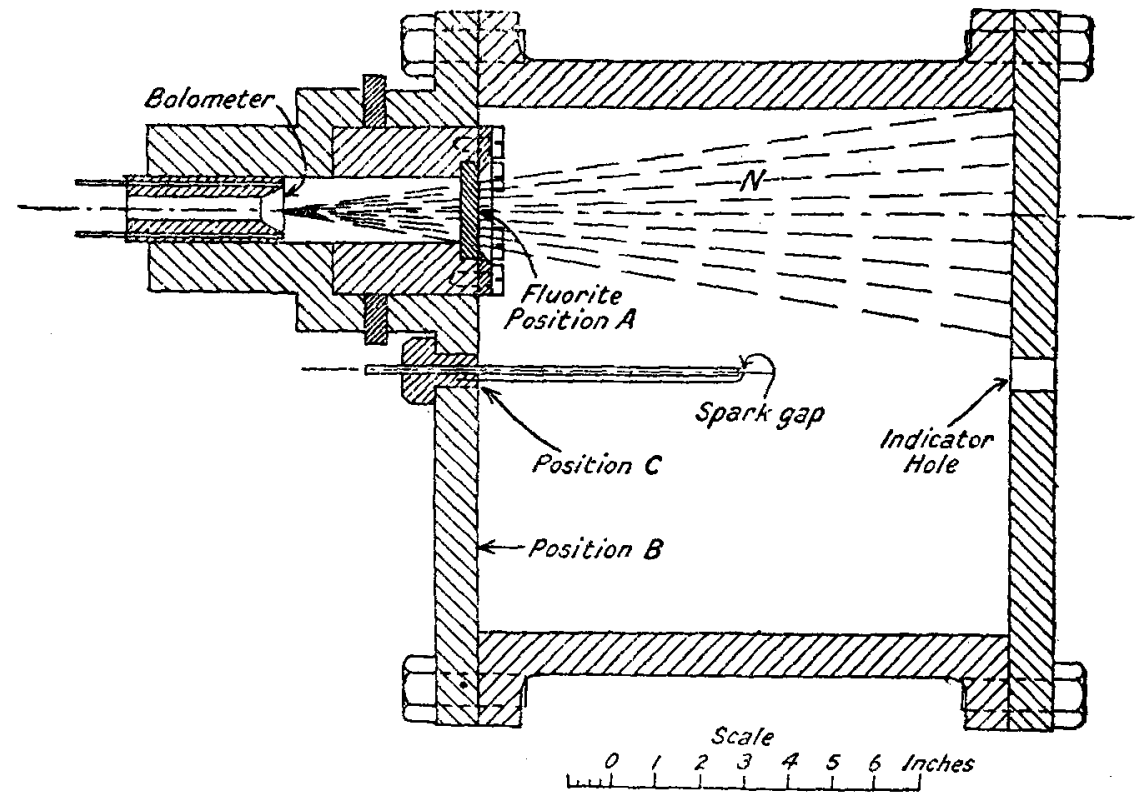

during explosion and subsequent cooling were measured by means of a Hopkinson optical indicator which threw a spot of light on to a revolving photographic film. The radiation was ineasured by means of a platinum bolometer connected with a reflecting galvanometer which also threw a spot of light on to the same revolving film. The bolometer was protected from the hot gaseous mixture by means of a plate of fluorite as shown in fig. 1 . The fluorite transmits almost exactly 95 per cent. of radiation of the wave-length emitted by an exploded coal-gas and air mixture. After making an allowance of 5 per cent. for the absorption of the fluorite and 5 per cent. for reflexion from the blackened surface of the bolometer, it is considered that the measurements from the films give radiation values of a high degree of accuracy. 
3. The radiation emitted was measured in three positions on one of the end-curves of the explosion vessel, viz. at the top (position A), at the bottom (position B), and at the centre (position $\mathrm{C}$ ). The radiation measured at position $\mathrm{O}$ was a little greater than that at either A or B ; and that measured at $A$ was a little greater than that at $B$. The mean value of the radiation received at $A, B$, and $C$ gives a fairly accurate estimate of the average radiation over the whole vessel.

4. When the bolometer was placed close up to the window of fluorite the radiation it received per sq. $\mathrm{cm}$. of its surface was equal to that received by a sq. $\mathrm{cm}$. of wall surface in the immediate neighbourhood; and when the bolometer was placed some distance away from the fluorite plate the radiation it received came from a cone of the gaseous mixture of small solid angle ( $N$, as shown in fig. 1). Throughout this paper the radiation measured with the bolometer in the latter position has been divided by the solid angle of the cone so as to give the radiation from a cone of unit solid angle. The rate of emission from a cone of gaseous mixture of unit solid angle has been called by Prof. Callendar the intrinsic radiance.

Fig. 2.

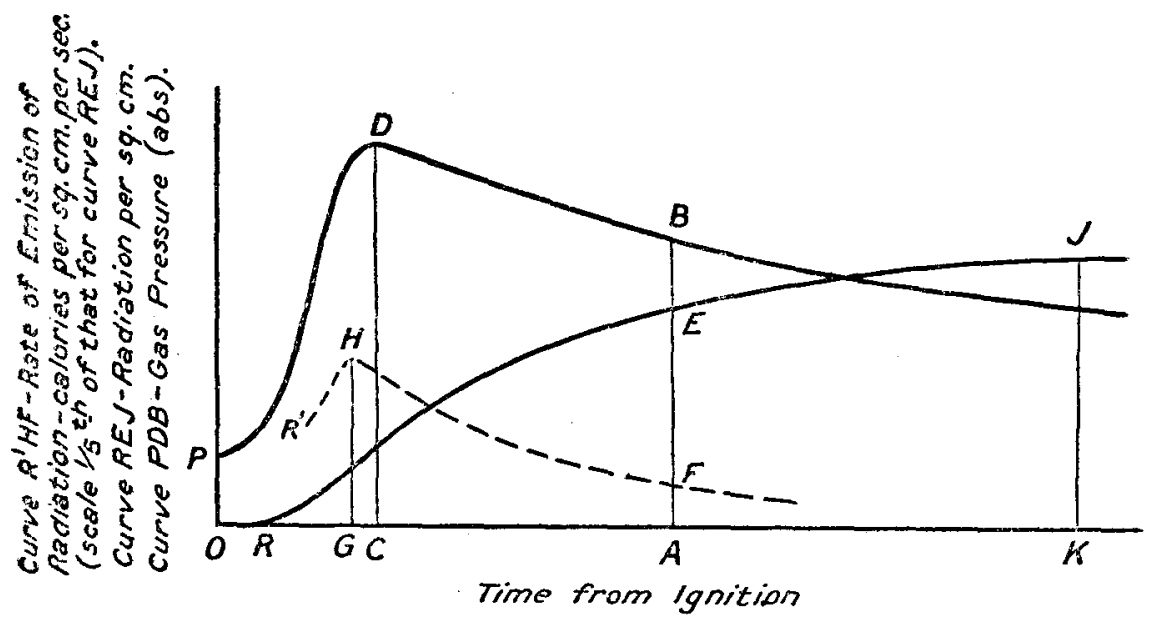

5. A list of the symbols used in this paper is given below. In defining them it will be convenient to refer to the curves in fig. 2, which have been talien from a typical record. 
$t$-time from ignition (OA).

$t_{e} \quad$-time of explosion (OG), i.e. time taken by gaseous mixture to develop its maximum pressure.

$p \quad$-pressure of gaseous mixture at time $t$ in $\mathrm{lb}$. per sq. in. (abs.) (AB).

$p_{\text {max. }}$-maximum pressure (CD).

$\hat{p} \quad$-rate of change of pressure at time $t$.

$\dot{p}_{\text {max. }}$ - the maximum rate of change of pressure (which occurs during the explosion peried).

$\theta \quad$-mean absolute temperature in ${ }^{\circ} \mathrm{C}$. of the gaseous mixture at time $t$ calculated from the pressure curve by means of the equation $p v=\mathrm{R} \theta$ after making a small correction (of the order of 3 per cent.) for contraction of volume which occurs on the combustion of the coal-gas.

$\vartheta_{\text {max. }}$-maximum mean absolute temperature developed in the gaseous mixture.

$\dot{\theta}$-rate of change of mean gas temperature at time $t$.

$\dot{\theta}_{\text {max. }}$-maximum rate of change of mean gas temperature (which occurs during the explosion period).

Q -quantity of coal-gas present in explosion vessel (measured in litres at atmospheric temperature and pressure).

" - volume of cylindrical vessel in c.c. of dimensions $l \mathrm{~cm}$. diameter and $l \mathrm{~cm}$. long $=\frac{\pi}{4} l^{3}$. (In the explosion vessel used in the experiments $l=30$.)

$a$-area of interior surface of vessel in sq. $\mathrm{cm}$. (In the explosion vessel used in the experiments $a=4380 \mathrm{sq} . \mathrm{cm}$.)

D - density of gaseous mixture in atmospheres.

$\mathrm{R}$ - total radiation received by walls of explosion vessel per sq. cm. of surface at time $t$ measured in calories ( $\mathrm{AE}$, when bolometer is close up to fluorite window).

$R_{T} \quad$-final value of $R$, i. e. the value of $R$ registered after a time when the gaseous mixture has cooled to such an extent that it emits no further radiation (KJ). For all practical purposes this is the radiation registered at 1 sec. after ignition, for after this time the radiation emitted was insignificant. 
$\dot{\mathrm{l}}$-differential coefficient of $\mathrm{R}$ with respect to $t$, $i . e$. the rate at which the walls receive radiation per sq. cm. of surface (AF).

$\dot{\mathrm{R}}_{\mathrm{max}}$ - the maximum rate at which the walls receive radiation per sq. cm. of surface $(H G)$. This takes place during the explosion period.

$\mathrm{R}_{2} \quad$-total radiation at time $t$ received by the bolometer per sq. cm. of its surface from a cone of gaseous mixture of unit solid angle (AE, when bolometer is some distance away from fluorite window).

$\mathrm{R}_{i \mathrm{~T}}$-final value of $\mathrm{R}_{i}$, i.e. the value of $\mathrm{R}_{i}$ registered after a time when the gaseous mixture has cooled to such an extent that it emits no further radiation $(\mathrm{KJ})$. For all practical purposes this is the value of $R_{i}$ registered at 1 second after ignition.

$\dot{\mathrm{R}}_{i} \quad$-differential coefficient of $\mathrm{R}_{i}$ with respect to $t$. This is the intrinsic radiance $(\mathrm{AF})$.

$\dot{\mathbf{R}}_{i \text { nux. }}$ - the maximum value of $\dot{\mathrm{R}}_{i}$ which occurs during the explosion period ( $\mathrm{HG}$ ).

\section{The Maximum Rate of Emission of Radiation during Explosion.}

(a) Cylindrical Explosion Vessel $30 \mathrm{~cm}$. diameter and $30 \mathrm{~cm}$. long.

6. It has previously been shown * that the rate at which the gaseous mixture emits radiation is a maximum some little time before the attainment of maximum pressure. From an examination of a large number of films it would appear that the maximum rate of emission occurs at or near the second point of inflexion in the pressure curve during the explosion period. The curves in fig. 3 have been taken from three typical films. The A curves relate to a 10.5 per cent. mixture of coal-gas and air at atmospheric density; the $B$ curves to a 10.2 per cent. mixture at 1.24 atmospheres: density; and the $C$ curves to a $9 \cdot 2$ per cent. mixture at 1.37 atmospheres densityt. The dotted curves are the difforentials of the radiation curves, and they show clearly that the maximum rate of emission occurs near the time when the gas-pressure or temperature curves undergo their second

* Phil. Trans. A. vol. cexi. p. 381.

+ All the gaseous mixtures referred to in this paper were originally at atmospheric temperature. 
inflexion, which, of course, takes place immediately after the period during which the rate of rise of gas pressure or temperature is a maximum.

Fig. 3.

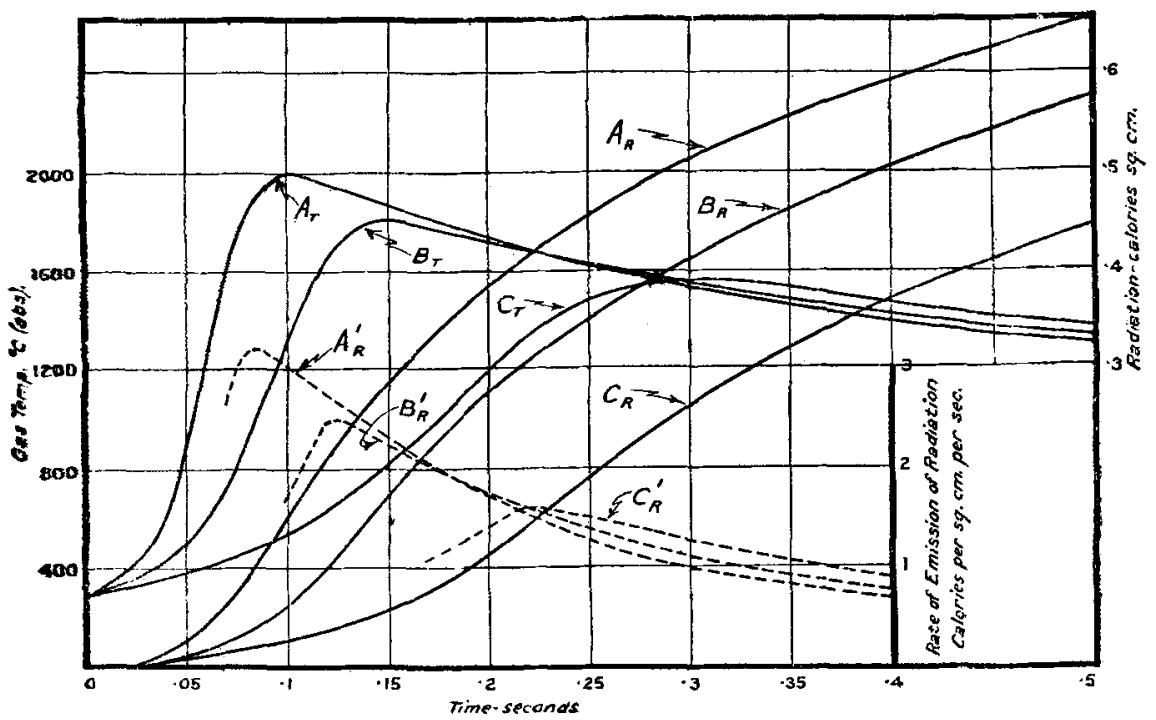

A curves : $105 \%$ mixture of coal-gas and air at atmospheric density.

B curves : $10 \% 2 \%$ mix 1 ure of conl-gns and air at $1 \cdot 24$ atmospheres density.

C curves: $9.2 \%$ mixture of coal-gas and air at 1.37 atmospheres density.

7. It occurred to the writer, therefore, to make an attempt to connect the maximum rate of emission $\left(\dot{\mathrm{R}}_{\max }\right)$ with the maximum rate of rise of pressure $\left(\dot{p}_{\max }\right.$. ) or of temperature $\left(\dot{\theta}_{\text {max. }}\right)$, and it was discovered that for mixtures containing the same quantity $(Q)$ of coal-gas $\dot{R}_{\text {max. }}$ was proportional to the square root of $\dot{\theta}_{\text {max. }}$. This will be seen on comparing columns 1 and 9 in Table $\mathrm{I}$. It will be noticed that the ratio $\frac{\dot{\mathbf{R}}_{\max }}{\sqrt{\dot{\theta}_{\max }}}$ is practically the same in all cases where $Q$ has the same value. Further, this ratio increases as $Q$ increases and it seems to be very approximately yroportional to the square root of $\mathrm{Q}$. The tigures in colnmn 10 are the ratios of the figures in column 9 to $\sqrt{ } Q-i . e$. they are the ratio

$$
\frac{\dot{\mathrm{R}}_{\max .}}{\sqrt{\mathrm{Q} \times \sqrt{\dot{\theta}_{\text {max. }}}}}
$$




\begin{tabular}{|c|c|c|c|c|c|}
\hline $\mid$ & $\begin{array}{l}\stackrel{i}{1} \\
\stackrel{g}{x} \\
\stackrel{x}{o} \\
\stackrel{\rho}{g}\end{array}$ & $\underset{-}{\sigma}$ & 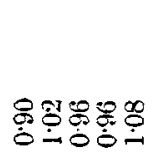 & 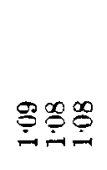 & $\hat{\dot{\theta}}$ \\
\hline | & $\begin{array}{ll}\stackrel{i}{i} & \\
\stackrel{0}{x} & \\
\stackrel{\leftrightarrow}{-} & 2 \stackrel{-}{-}\end{array}$ & $\stackrel{\varphi}{-}$ & 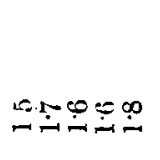 & ํㅗㄴ영영 & $\stackrel{9}{9}$ \\
\hline . & $\stackrel{9}{\dot{\theta}}$ & $\stackrel{\sim}{\infty}$ & 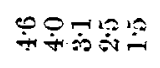 & 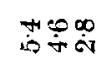 & 9 \\
\hline$e^{\infty}$ & 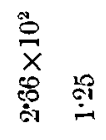 & 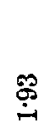 & $\begin{array}{l}\hat{0} \\
0 \\
0 \\
0\end{array}$ & 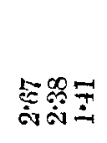 & $\stackrel{\infty}{\stackrel{\circ}{\sim}}$ \\
\hline 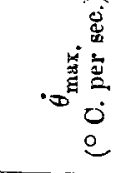 & $\begin{array}{l}\stackrel{\overrightarrow{0}}{\mathscr{D}} \\
\times \\
\overrightarrow{\mathbf{E}}\end{array}$ & $\underset{\mathfrak{c}}{\mathfrak{N}}$ & 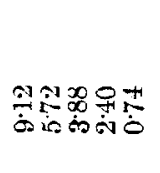 & 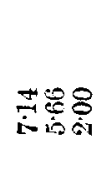 & iv \\
\hline 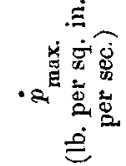 & $\underset{\substack{\infty \\
-1}}{8}$ & $\stackrel{8}{8}$ & 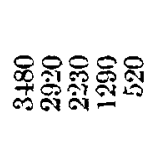 & 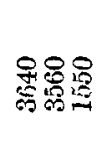 & $\begin{array}{l}8 \\
8 \\
0 \\
0\end{array}$ \\
\hline$\sum^{10}$ & $\stackrel{P}{\stackrel{N}{-}}$ & $\stackrel{9}{\stackrel{9}{9}}$ & 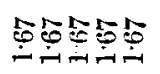 & $\stackrel{\infty}{\infty} \underset{\sim}{\infty}$ & 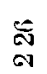 \\
\hline 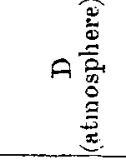 & $19 \stackrel{0}{0}$ & $\stackrel{\circ}{-}$ & $\begin{array}{l}19 \\
0\end{array}$ & 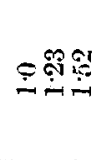 & 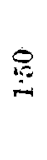 \\
\hline 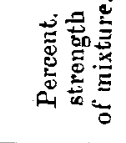 & 最 & $\stackrel{15}{9}$ & 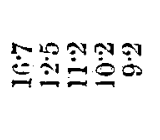 & 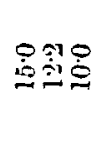 & : \\
\hline 禺离 & $\stackrel{-}{\dot{\varphi}} \overline{\dot{\Theta}}$ & $\begin{array}{l}0 \\
\stackrel{0}{0}\end{array}$ & 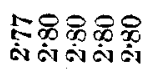 & 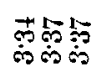 & $\bigodot_{i=0}$ \\
\hline
\end{tabular}


for the various mixtures (which it should be noticed vary in density as well as in strength). These ratios are all pretty much the same-indeed, having regard to the large variations in $\dot{\mathrm{K}}_{\text {max. }}, \dot{\theta}_{\text {max. }}, \mathrm{Q}$, and $\mathrm{D}$ it is somewhat surprising to find them agree with one another so closely.

8. It would therefore appear that certainly within the limits of mixture strength $9 \cdot 2$ per cent. to 15 per cent. ${ }^{*}$ and of density $\frac{1}{2}$ atmosphere to a little over $1 \frac{1}{2}$ atmospheres (as shown in Table I.) $\dot{\mathrm{h}}_{\text {max. }}$ in the explosion vessel used in the experiments is proportional to the square root of the product $\mathrm{Q} \times \dot{\theta}_{\text {max. }}$ Expressed in the form of an equation

$$
\dot{\mathrm{R}}_{\text {max. }}=\mathrm{K} \sqrt{\mathrm{Q} \times \dot{\theta}_{\text {max. }}}, . . . .
$$

where $K$ is a constant equal to $1 \cdot 0 \times 10^{-2}$ or $0 \cdot 01$, i.e. the mean value of the figures in column 10 .

It may be noticed that as $\dot{\theta}$ is proportional to $\frac{\dot{p}}{\mathrm{D}}$ (the ratio $\frac{\dot{\theta} \times \mathrm{D}}{\dot{p}}$ being equal to $19 \cdot 6$ ) this equation may be written

$$
\dot{\mathrm{R}}_{\text {max. }}=\mathrm{K}_{1} \sqrt{\frac{\mathrm{Q} \times \dot{p}_{\text {max. }}}{\mathrm{D}}}, \ldots .
$$

where $K_{1}=0 \cdot 01 \times \sqrt{19 \cdot 6}=0 \cdot 0445$.

9. Some confirmation is lent to the proportionality between $\dot{\mathrm{R}}_{\text {max. }}$ and $\sqrt{\mathrm{Q} \times \dot{\theta}_{\text {max. }}}$ from the writer's measurements of the intrinsic radiance of coal-gas and air mixtures at various densitjes from $\frac{1}{2}$ atmosphere to $1 \frac{1}{4}$ atmospheres $\dagger$. These are set out in Table II. (which contains similar information to that given in 'lable I.). It will be noticed that the ratios in column 10 are again very much the same, though of course they differ from those in column 10 in Table $I$. because the values of $\dot{\mathrm{R}}_{i \text { max. }}$ are different from those of $\dot{\mathrm{R}}_{\text {max. }}$. The equations for the maximum intrinsic radiance are therefore

$$
\begin{aligned}
\dot{\mathrm{K}}_{i \max } & =0.0117 \sqrt{\mathrm{Q} \times \dot{\theta}_{\max .}} . . . \\
\text { and } \quad \dot{\mathrm{R}}_{i_{\text {max. }}} & =0.052 \sqrt{\frac{\mathrm{Q} \times \dot{p}_{\max .}}{\mathrm{D}} .} . .
\end{aligned}
$$

* A 15 per cent. mixture is the strongest that will burn completelyi.e. it uses up the whole of the oxygen of the air. The weakest mixture that will explode is probably between 7 per cent. and 8 per cent. The limits of mixture strength in these experiments are therefore very wide. + Phil. Trans. A. vol. cexi. p. 395. 


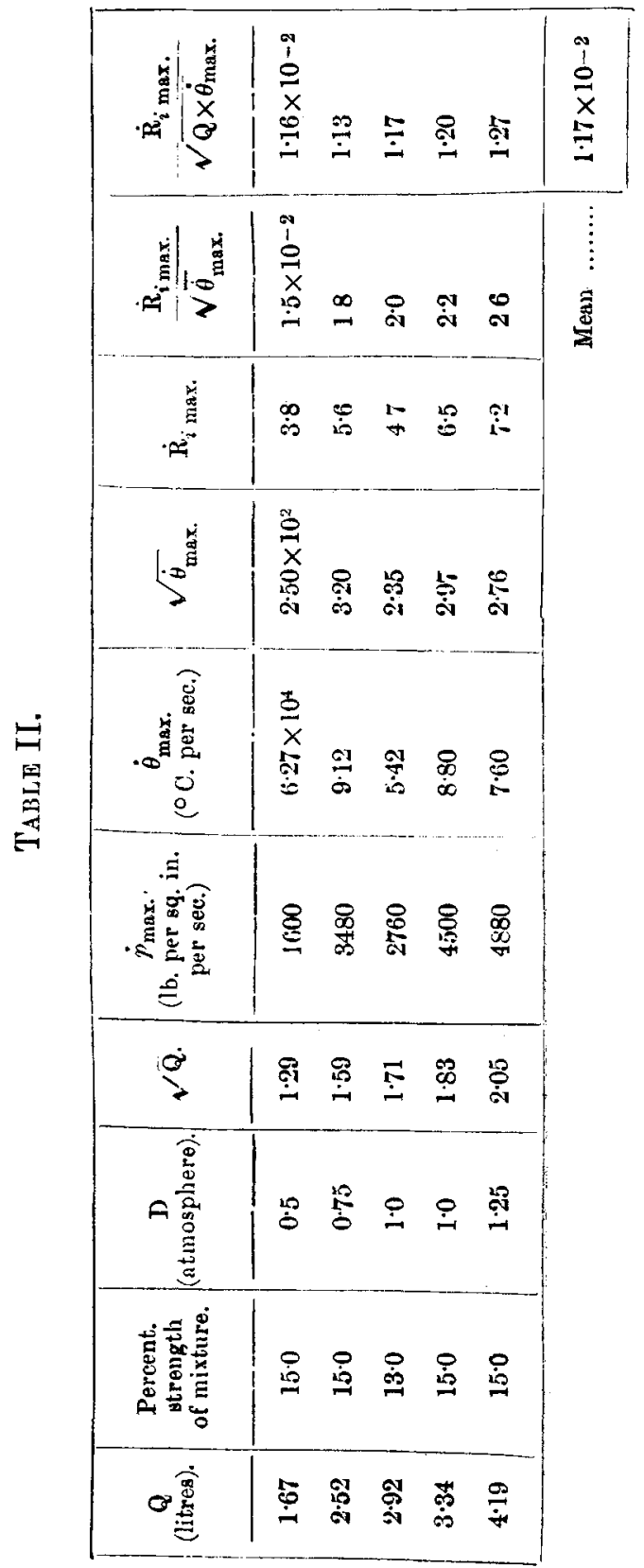


10. In considering these formula it is suggestive to regard $Q$ as being proportional to the density of distribution of chemical energy in the inflammable mixture and $\dot{\theta}$ as being proportional to the rate of conversion of chemical energy into thermal energy. In a previous paper it has been suggested that the chemical energy in process of conversion first passes (either wholly or a consideralle proportion of it) into the form of internal vibrations of the combining molecules, and it is reasonable to suppose that the radiation to which these vibrations give rise and which comes from the whole mass of the burning gas should be a maximum towards the end of the period during which combustion proceeds at its maximum rate.

\section{(b) Cylindrical Explosion Vessel l $\mathrm{cm}$. diameter and $\mathrm{l} \mathrm{cm}$. long.}

11. It will be realized that the radiation values in the preceding sections have referred to explosions in a single vessel $30 \mathrm{~cm}$. in diameter and $30 \mathrm{~cm}$. long. Some indication of the effect of size of vessel on the radiation values may, however, be obtained from the writer's experiments on the diathermancy of coal-gas and air mixtures exploded in a vessel with silverplated walls which could be made reflecting by polishing or absorbent as regards radiation received by them by coating with a thin layer of dull black paint *

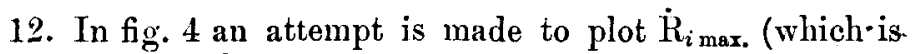
proportional to $\dot{\mathrm{R}}_{\text {max. }}$ ) for mixtures of various densities for a cylindrical vessel $l \mathrm{~cm}$. diameter and $l \mathrm{~cm}$. long against $l$. Confine the attention first to the thick curve which connects $\dot{\mathrm{R}}_{i \max }$ with $l$ for a $1 \tilde{\mathrm{j}}$ per cent. mixture at atmospheric density. The value for $l=30$ (viz. 6.6 calories per sq. $\mathrm{cm}$. per sec.) is, of course, directly obtained from the experiments, for the cylindrical vessel used in these experiments was of dimensions $30 \mathrm{~cm}$. by $30 \mathrm{~cm}$. It is clear, too, that $\dot{\mathrm{R}}_{i}=0$ for $l=0$, so that there are two definite points on the curve. Two other points, one for $l=15$ and the other for $l=59$, miny be roughly fixed in the light of the following considerations. That for $l=59$ will certainly lie somewhere between $\dot{\mathrm{R}}_{i \max }$ for $59 \mathrm{~cm}$. in the polished vessel (which is 10.9) and $\dot{\mathrm{R}}_{i \text { max. }}$ for $59 \mathrm{~cm}$. in the blackened vessel (which is 8.4 )

* Phil. Trans. A. vol. cexi. p. 395. 
and probably will lie rather nearer the latter*. The other for $l=15$ will in all probability lie between $\dot{\mathrm{R}}_{i \max }$. from $15 \mathrm{~cm}$. in the blackened vessel (which is $4 \cdot 7$ ) and half that from $30 \mathrm{~cm}$. in the blackened vessel (which amounts to $3 \cdot 3) \uparrow$.

Fig. 4.

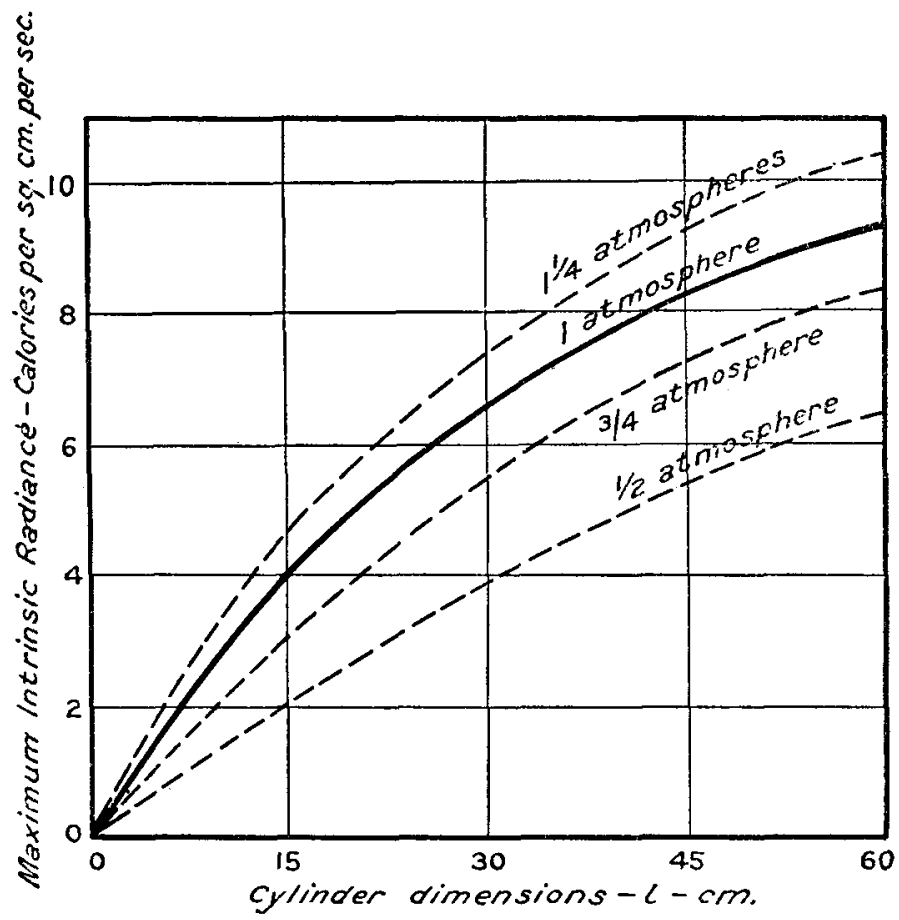

The thick line then is a fair curve drawn through the two definite points $(0,0)$ and $(30,6 \cdot 6)$ and between the two pairs of points $(15,3 \cdot 3-4 \cdot 7)$ and $(59,8 \cdot 4-10 \cdot 9)$.

* There seems little doubt that the radiation from a layer of gas increases not only with its thickness but with its lateral dimensions as well. The radiation from a cylindrical mass of gas $59 \mathrm{~cm} . \times 59 \mathrm{~cm}$. will therefore be greater than that from a cylindrical mass $59 \mathrm{~cm}$. long and $30 \mathrm{~cm}$. in diameter. On the other hand, the radiation from $59 \mathrm{~cm}$. in the polished vessel approximates to that from a mass of gas $59 \mathrm{~cm}$. in thickness but of infinite lateral dimensions.

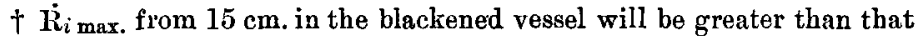
from a cylindrical mass $15 \mathrm{~cm}$. by $15 \mathrm{~cm}$., because in the former case the lateral dimensions are greater than in the latter. 
The dotted curves relate to 15 per cent. mixtures at $\frac{1}{2}$ atmosphere, $\frac{3}{4}$ atmosphere, and $1 \frac{1}{4}$ atmospheres density, and they have been drawn from similar data.

It is not suggested that these curves give a very accurate relationship between $\dot{\mathrm{R}}_{i \text { max. }}$ and cylinder dimensions, but it is considered that they give a very definite indication of the shape of the true curves.

13. These curves (with the exception of that for $\frac{1}{2}$ atmosphere density) indicate that $\dot{\mathrm{R}}_{i \max }$ (and therefore $\dot{\mathrm{R}}_{\text {max. }}$ ) varies more or less as $\sqrt{l}$. The curve for $\frac{1}{2}$ atmosphere density is practically a straight line up to $l=30$. This seems reasonable in view of the known higher transparency of the gaseous mixture at this density.

Assuming the relationship $\dot{\mathrm{R}}_{\text {max. }} \propto \sqrt{\bar{l}}$ to be reasonably near the truth, equations (1) and (2) may be amended to. cover varying volume in the following way :-

$$
\begin{aligned}
\dot{\mathrm{R}}_{\text {max. }} & =0.01 \sqrt{\frac{l}{30} \sqrt{\mathrm{Q} \times \dot{\theta}_{\text {max. }}}} \\
& =0.00183 \sqrt{l \times \mathrm{Q} \times \dot{\theta}_{\text {max. }}} . . .
\end{aligned}
$$

And equations (2), (3), and (4) will become-

$$
\begin{aligned}
& \dot{\mathrm{R}}_{\text {max. }}=0.00812 \sqrt{\frac{l \times Q \times \dot{p}_{\text {max. }}}{\mathrm{D}}, \ldots .} \\
& \dot{\mathrm{R}}_{i_{\text {max. }}}=0.00214 \sqrt{l \times Q \times \dot{\theta}_{\text {max. }}}, \ldots . \\
& \dot{\mathrm{R}}_{i_{\text {max. }}}=0.0095 \sqrt{\frac{l \times Q \times \dot{F}_{\text {max }}}{\mathrm{D}}} . . .
\end{aligned}
$$

14. These equations it is thought may be expected to give values for $\dot{\mathrm{R}}_{\max }$, and $\dot{\mathrm{R}}_{i \max }$ correct to within perbaps 10 per cent. when applied to mixtures of coal-gas and air ranging from 9 to 15 per cent., within the limits of density $\frac{3}{4}$ atmosphere to perbaps $1 \frac{1}{2}$ or 2 atmospheres and within the limits of cylinder dimensions $15 \mathrm{~cm} . \times 15 \mathrm{~cm}$. to $60 \mathrm{~cm} . \times 60 \mathrm{~cm}$.

Further experimental work will be required in order to decide whether they apply approximately outside these limits. 
Rate of Emission of Radiation during (ooling.

(a) Cylindrical Explosion Vessel $30 \mathrm{~cm}$. diameter and $30 \mathrm{~cm}$. long. Gaseous Mivtures at Atmospheric Density.

15. It is not possible to build up an accurate formula for $\dot{R}$ at any temperature under wide conditions of mixture strength, density, and volume until much further experimental work is accomplished. The whole question is complicated greatly by the fact that the transparency of the gaseous mixture varies with the time after ignition, and also (to a much less extent) because the vibratory energy of the molecules is dependent upon other factors as well as temperature*. It is important, however, from the point of view of gas-engine calculations to be able to estimate the radiation loss at various points during the expansion stroke, and it is believed that an empirical formula sufficiently accurate for this purpose can be established.

16. It has been previously shown that the radiation from the gaseous mixture when corrected for absorption varies with the temperature approximately in accordance with Planck's formula for a wave-length of $3 \cdot 6 \mu \dagger$ (which at high temperatures varies very nearly as the square of the absolute temperature). It so happens, however, that when uncorrected for absorption $\dot{R}$ in a vessel of the dimensions used in the experiments is approximately proportional to $\theta^{4}$, and it is considered that a formula based upon this law will give gasengine designers fairly reliable information as to radiation loss when applied to cylinders within the limits of dimensions $15 \mathrm{~cm} . \times 15 \mathrm{~cm}$.to $60 \mathrm{~cm} . \times 60 \mathrm{~cm}$.

* See Phil. Mag. Feb. 1913, p. 267.

† Phil. Trans. A. vol. cexi. p. 402.

I See Phil. Trans. p. 386 , fig. 9. The $\theta^{t}$ curre there was made to coincide with $i \vec{i}$ at comparatively low gas temperatures when the radiation is small. By increasing the ecale a close agreement may be obtained at the high temperatures $\left(2400^{\circ} \mathrm{C}\right.$. abs. to $1600^{\circ} \mathrm{C}$. abs.), which is the important part of the curve from the point of view of the gas-engine. The agreement between the $\theta^{4}$ curve and $\dot{R}$ in the neighbourhood of the maximum temperature in the 9.8 per cent. mixture is not so good, but a fairly correct value for $\dot{R}$ in this epoch for weak mixtures may be obtainer by calculating the mean of $\dot{R}$ given by the $\theta^{4}$ formula (equation (9) $\S 17$ ) an $\mathrm{d} \dot{\mathrm{K}}_{\text {max. }}$ as determined by the equation in the preceding sections.

The statement made in the paper referred to (footwote p. 402) to the effect that the $\theta^{2}$ law would not hold in the case of a cylinder of widely different dimensions from those of the vessel cised in the experiments requires some modification. There seems little doubt that this statement 
17. In a vessel $30 \mathrm{~cm}$. in diameter and $30 \mathrm{~cm}$. in length

$$
\dot{\mathrm{R}}=1 \cdot 75 \times 10^{-14} \theta^{4} \text {, . . . . }
$$

where the constant $1.75 \times 10^{-14}$ has been determined from the writer's experiments *.

(b) Effect of Density and of Cylinder Dimensions.

18. These experiments also indicate that $\dot{R}$ varies as $\sqrt{\bar{D}}+$, and as we have seen in $\S 13$ of this paper it also varies as $\sqrt{ } l$.

In order to cover density and cylinder dimensions equation (9) thus becomes

$$
\begin{aligned}
\dot{\mathrm{R}} & =1.75 \times 10^{-14} \theta^{4} \sqrt{\mathrm{D}} \sqrt{\frac{l}{30}} \\
& =0.32 \times 10^{-14} \theta^{4} \sqrt{l \times \bar{D}} . . .
\end{aligned}
$$

19. This equation has been used to calculate $\dot{\mathrm{R}}$ in a large number of cases for the ressel $30 \mathrm{~cm}$. by $30 \mathrm{~cm}$. within the limits of mixture strength $9 \cdot 2$ to 15 per cent. and of density $\frac{1}{2}$ atmosphere to $1 \frac{1}{2}$ atmospheres, and it has been found that the calculated value agrees with the observed value to within 15 per cent.

20. It will be clear that equations (1) to (10) apply only in the case of a cylindrical vessel whose length is equal to its diameter. In a gas-engine, however, the length varies throughout the stroke, and some modification of these equations is necessary in order that they may be applied directly to gas-engine calculations. This problem will be considered in another paper.

is true in the case of a vessel whose length is varied but whose diameter remains fixed at $30 \mathrm{~cm}$. (Compare curves $C$ and $I$, fig. 14, p. 396.) When, however, the diameter is varied in the same proportion as the length it is probable that, owing to the fact that the transparency varies with the lateral dimensions; $\dot{\mathrm{h}}$ would more or less follow the $\theta^{1}$ law. (Compare curves $\mathrm{A}$ and $\mathrm{D}$ in the same figure. The former gives the intrinsic radiance for a cylindrical mass of gas of effective length $59 \mathrm{~cm}$. and of effective diameter greater than $59 \mathrm{~cm}$., while the latter gives the intrinsic radiance for a cylindrical mass $30 \mathrm{~cm}$. in diameter and $30 \mathrm{~cm}$. long. Both curves follow the $\theta^{2}$ law approximately.)

* Phil. Trans. A. vol. cexi. p. 386, fig. 9.

$\dagger$ Phil. Trans. A. vol. ccxi. pp. $388 \& 405$. 


\section{Total Radiation emitted during Explosion and Subsequent Cooling.}

(a) Cylindrical Vessel $30 \mathrm{~cm}$. diameter and $30 \mathrm{~cm}$. long; Gaseous Mixtures at Atmospheric Density.

21. In fig. 5 are given mean gas temperature curves and total radiation curves per sq. $\mathrm{cm}$. of wall surface for mixtures of coal-gas and air of atmospheric density varying in strength from $9 \cdot 7$ per cent. to 15 per cent. The radiation curves were all taken when the bolometer was in position $\mathrm{C}$, in which

Fig. 5.

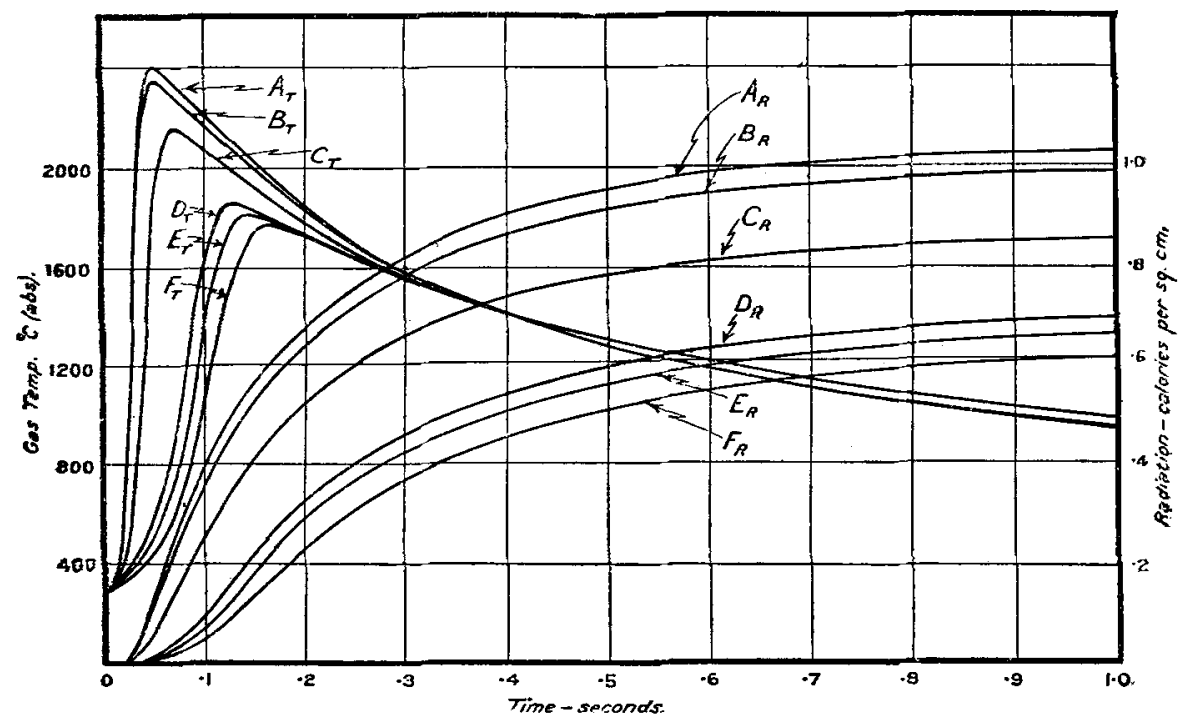

A curves: $15 \%$ mixture of coal-gas and air.

B curres: $15 \%$ mixture,$\quad$ (taken at a later date when the cal. value of the conl-gas had probably altered).

C curves: $13 \%$ mixture of coal-gas and air.

D curves: $10.2 \%$ mixture " "

E curves: $10.0 \%$ mixture,$"$,

F curves: $9.8 \%$ mixture " ,

position the radiation measured was very approximately 5 per cent. greater than the mean of that measured in positions $\mathrm{A}, \mathrm{B}, \& \mathrm{C}$. The curves cover a period of one second after ignition, after which time the radiation from the gaseous mixture is very small. 
22. A glance at these curres shows at once that a close relationship exists between the total radiation emitted and the maximum gas temperatures developed in the various mixtures, and in fig. 6 the total radiation received by the walls per sq. cm. * has been plotted against the maximum gas temperatures. It will be noted that the total radiation is a linear function of the maximum temperature. The equation to this line is

$$
\mathrm{R}_{\mathrm{T}}=0.00056\left(\theta_{\text {max. }}-700\right) . . . .
$$

(b) Variation of Total Radiation with Hensity and Cylinder Dimensions.

23. The writer does not possess sufficient records to enable him to plot similar curves to that in fig. 6 for other densities.

Fig. 6.

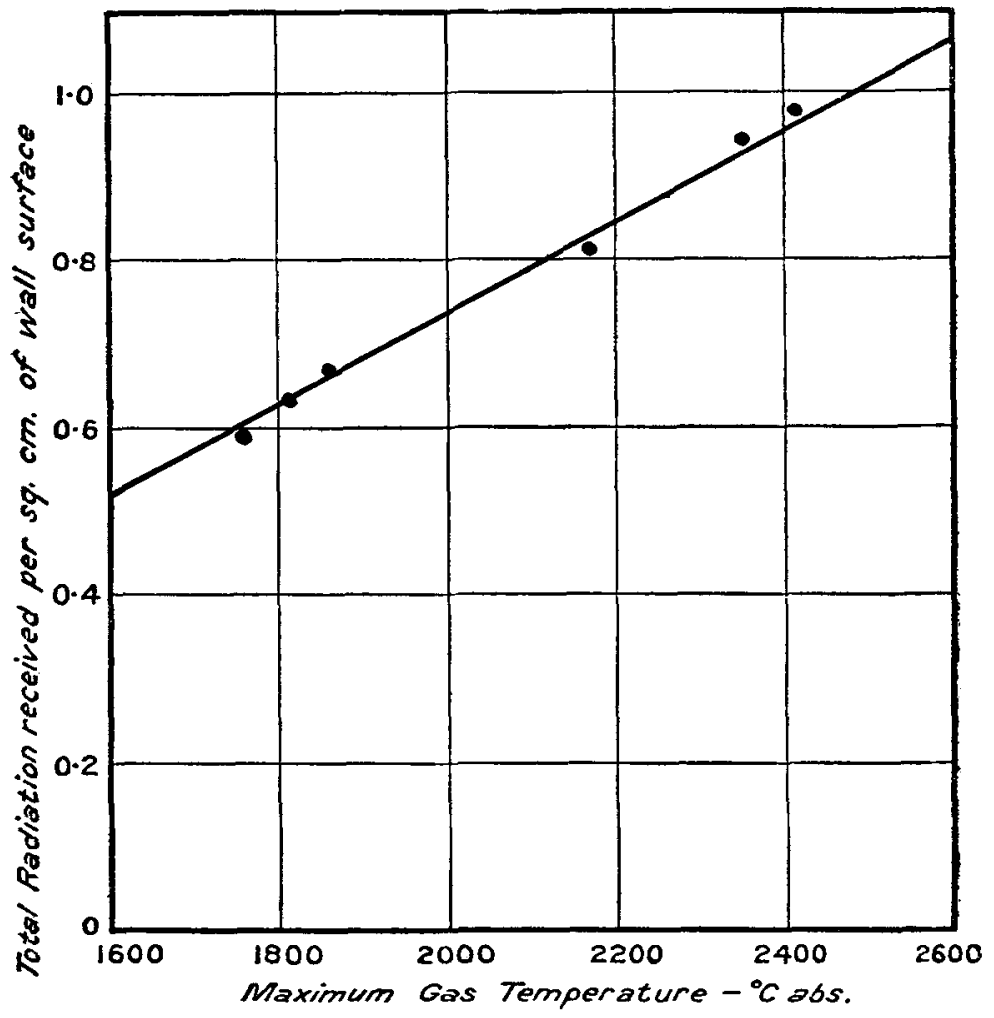

* The radiation values in fig. 6 are those shown in fig. 5 multiplied by 0.95 so as to give the mean values over the whole vessel.

Phil. Mag. Ser. 6. Vol. 39. No. 229. Jan. 1920. G 
He has, however, two pairs of records for various mixture strengths ${ }^{*}$, the one pair at $1 \frac{1}{4}$ atmospheres density and the other pair at $1 \frac{1}{2}$ atmospheres density. Calculations from these records give the information shown in Table III.

\begin{tabular}{cccc}
\multicolumn{4}{c}{ TabLe III. } \\
$\begin{array}{ccc}\text { D } \\
\text { (atmospheres). }\end{array}$ & $\begin{array}{c}\text { Percent. strength } \\
\text { of mixture. }\end{array}$ & $\theta_{\text {max. }}$ & $\mathbf{R}_{\mathrm{T}^{*}}$ \\
1.24 & $10 \cdot 2$ & 1800 & 0.7 \\
1.24 & $12 \cdot 2$ & 2250 & 0.94 \\
1.50 & $15 \cdot 0$ & 2400 & $1 \cdot 17$ \\
1.56 & $10 \cdot 0$ & 1840 & 0.87
\end{tabular}

The equation derived from the first pair at $1 \frac{1}{4}$ atmospheres density is

$$
\mathrm{R}_{\mathrm{T}}=0.00053\left(\theta_{\text {max. }}-480\right), \ldots . \quad .
$$

and that from the second pair at $1 \frac{1}{2}$ atmospheres density (approximately) is

$$
\mathrm{R}_{\mathrm{T}}=0.00053\left(\theta_{\max }-190\right) . . . .
$$

24. In considering these equations it must be remembered that each is derived from two points only and the constants are therefore not to be relied upon. They show fairly definitely, however, that the greater the density the smaller the constant within the brackets, and it would appear that this constant is dependent upon the shape of the cooling curve.

25. An examination of the records for 15 per cent. mixtures shows that between the limits of density $\frac{3}{4}$ atmosplere and $1 \frac{1}{2}$ atmospheres $R_{\mathrm{T}}$ and $R_{i \mathrm{~T}}$ vary approximately as $\sqrt{ } \mathrm{D}$. An approximate relationship between $\mathrm{R}_{\mathrm{T}}, \theta$, and $\mathrm{D}$ is therefore given by

$$
R_{\mathrm{T}}=0.00056\left(\theta_{\text {max. }}-700\right) \sqrt{\mathrm{D}} . . .
$$

* Taken in position A.

$\dagger$ The form of equations (12) and (13) indicates that it world have been better to express equation (14) in the form $R_{T}=0 \cdot 00055\left[\theta_{\max }-700 f(D)\right]$ where $f(\mathrm{D})=\frac{480}{700}$ when $\mathrm{D}=1.24$ or $\frac{190}{700}$ when $\mathrm{D}=1.53$. But as has been stated equations (12) and (.3) have been derived from two pairs of 
26. These records also show that $\mathrm{R}_{i \mathrm{~T}}$ varies with cylinder dimensions in a similar way to that of $\dot{\mathrm{R}}_{2}$ (see $\S 13$ ). For purposes of rough calculation we may therefore write

$$
\begin{aligned}
\mathrm{R}_{\mathrm{T}} & =0.00056\left(\theta_{\max }-700\right) \sqrt{\mathrm{D}} \sqrt{\frac{l}{30}} \\
& =0.0001\left(\theta_{\max }-700\right) \sqrt{\mathrm{D} \times l} . . . .
\end{aligned}
$$

\section{Summary.}

27. An attempt has been made in this paper to build up formulæ by means of which the emission of radiation during explosion and subsequent cooling of inflammable mixtures of coal-gas and air of various strengths in a cylindrical vessel whose diameter is equal to its length and whose walls are black may be estimated from the pressure time-curres for these mixtures. An attempt has also been made to introduce factors into these formulæ so that the radiation may be estimated roughly when the density is other than atmospheric, and also when the explosive gaseous mixture is contained in a cylindrical vessel of dimensions different from those of the experimental vessel.

The conditions governing the emission of radiation in gaseous explosions are exceedingly complex and the formula set up can only be relied upon to give results correct to within from 10 to 20 per cent. Nevertheless as no such formula lave hitherto been set up their formulation it is considered will serve a useful purpose in that they may be used as a basis for gas-engine calculations.

points only, and consequently the figures 480 and 190 may not be sufficieutly reliable to enable one to evaluate $f(D)$ with any degree of accuracy. In view of the fact that equation (14) gives results which are fairly well in accord with values for $R_{r}$ found by experiment within the limits of density $\frac{3}{4}$ atmosphere and $1 \frac{1}{2}$ atmospheres, it has been thought worth while to include it in this paper. 